\title{
Analyzing the Duality Concept through an Entropy Approach
}

\author{
S. Abdelhady ${ }^{*}$, M.S. Abdelhady ${ }^{\dagger}$
}

\begin{abstract}
According to a previously introduced entropy approach; the flow of electric charges was defined as a flow of electromagnetic waves that has an electric potential. Similarly; the magnetic flux was defined as a flow of electromagnetic waves that has a magnetic potential. By a similar entropy approach, particle's kinetic energy is defined also as electromagnetic waves. So: the electron can be defined as an energized particle whose electric charge, magnetic energy and kinetic energy are electromagnetic waves. According to such definition and to the found similarity of the mechanisms and laws of mass and energy diffusions in additions to the Einstein's principle of mass-energy equivalence; the flow of electrons can be postulated as a simultaneous flow of two energy components; particulized energy and wave energy. Hence; the electron hasn't a dual nature but its behavior as a particle or as wave depends on the relative values of its two components in each flow.

Truth of the introduced postulates is proved through finding also plausible explanation of the sintering phenomena and de-Broglie hypothesis.
\end{abstract}

\section{Introduction}

The propagation of waves through space is quite different from the propagation of particles. Flow of particles is governed by the laws of particle-mechanics while flow of waves is characterized by Maxwell's equations of electromagnetism [1]. However, the propagation of particles was confused as a flow of waves in some experiments and the flow of waves was confused also as a flow of particles in other experiments $[2,3]$. Such results led to introduce the concept of "wave- particle duality" that stated the matter and light may exhibit the behaviors of both waves and particles depending upon the circumstances of each experiment. In this article; such confusion is analyzed through an energy-entropy approach that defined the electric charge and magnetic flux as forms of energy or electromagnetic waves of definite values of electric or magnetic potentials $[4,5]$. Some references defined also the kinetic energy as a form of magnetic energy or electromagnetic waves [6, 7]. By following the considered entropy approach, the kinetic energy is found also as electromagnetic waves. Accordingly; the electron can be defined as an energized particle whose charge, magnetic and kinetic energies are forms of electromagnetic waves. According to the similarity of the mechanisms and laws that govern mass and energy diffusions in addition to the Einstein's principle of mass-energy equivalence [8]; it is possible to consider the flow of electrons as a simultaneous flow of two components belonging to two systems: a particulized-energy system and a wave-energy system. The relative values of the wave-energy and the particulized energy components define the electron's memberships to the two systems and describe the flow of electrons as a simultaneous flow of waves and particles. Such definitions find plausible

\footnotetext{
*Ph.D., South Valley University, Egypt.

${ }^{\dagger}$ Ph.D., BeniSouif University, Egypt.
} 
explanation of the duality property of electrons as discovered by the results of J.J. Thomson in the Cathode ray tube [9]. According to such postulate; it is possible to find also plausible explanation of the solid state sintering-phenomena [10]. During sintering; the bores between crystals of some refractory materials are filled by flow of thermal or electrical energy across the sintered materials at temperatures below its melting point. The flow of heat or charge cannot fill the bores by waves but such bores are filled, as postulated, by a simultaneous flow electromagnetic waves and particles. In other words; flow of waves, as heat or electric charges, should be simultaneously accompanied by a flow of particles to fill the sintered bores and this proves the truth of the introduced simultaneous-flow postulate.

The truth of such postulates is verified when reviewing other hypothesis as the de-Broglie hypothesis [11] and thermoelectricity [12].

\section{Electric Charge and Magnetic Flux}

In a previous study; the time $\tau$ was replaced by the entropy $\mathbf{s}$ in the Maxwell's wave equations and the electric charge was defined as a special solution of such Maxwell's equation that is represented as follows [4]:

$$
\begin{gathered}
E(r, s)=s(\varphi(r, s))+/-\Delta \bar{E} \\
H(r, s)=s(\varphi(r, s))
\end{gathered}
$$

The previous solution defines the flow of electric charges as a flow of ionized electromagnetic waves which have a specified value of non-zero electric potential " $+/-\Delta \overline{\mathbf{E}}$." The graphical representation of such solution at specific position of the dimensional coordinate $\mathbf{r}$ is seen in Figures 1 and $\mathbf{2}$ where the electric potential $\mathbf{E}$ of such waves is oscillating, in the $\mathbf{E}$-s plane, around an initial non-zero value. Figure 1 represents a positive charge or an ionized electromagnetic wave that has a net positive potential. Similarly, Figure 2 represents a negative charge or an ionized electromagnetic wave of negative potential. The charge in each imparted sinusoidal wave may have a net positive or a negative potential according to the sign of the following integral [5]:

$$
\mathrm{Q}_{\text {elect }}=\int_{0}^{2 \pi} \overline{\mathrm{E}} \mathrm{dS} \text { elect }
$$

Similarly; the following solution of the Maxwell's wave equations represent the magnetic flux as electromagnetic waves whose magnetic potential $\mathbf{H}$ is oscillating around a specified value of non-zero magnetic potential " $+/-\Delta \overline{\mathbf{H}}$ ” in the $\mathbf{H}-\mathbf{s}$ plane but its electric potential $\mathbf{E}$ is oscillating about a zero electric potential in the E-s plane [4].

$$
\begin{gathered}
\mathrm{H}(\mathrm{r}, \mathrm{t})=\mathrm{s}(\varphi(\mathrm{r}, \mathrm{t}))+/-\Delta \overline{\mathrm{H}}) \\
E(\mathrm{r}, \mathrm{t})=\mathrm{s}(\varphi(\mathrm{r}, \mathrm{t}))
\end{gathered}
$$

Such solution is represented graphically in Figure $\mathbf{3}$ where the net bounded area in the $\mathbf{H}-\mathbf{s}$ plane has a non-zero magnetic potential. So; the net value of the energy that has a magnetic potential or the magnetic flux has the flowing value [5]:

$$
\Phi=\int_{0}^{2 \pi} \bar{H} d S_{\text {mag }}
$$

Such postulates consider the electric charge and magnetic flux as energies that have specific potentials as the heat is considered as energy of specific thermal potential (temperature) [14]. 


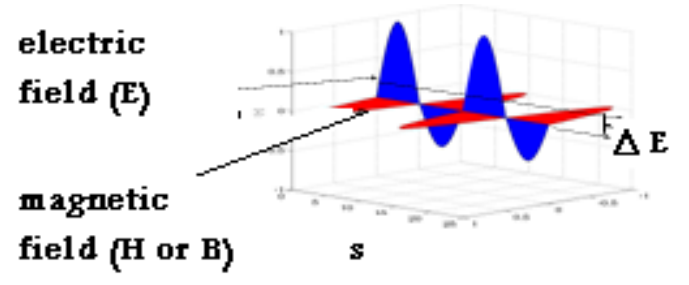

Fig.1. Graphical representation of a positive electric charge

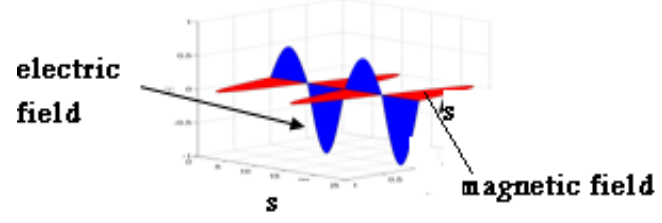

Fig.2. Graphical representation of a negative electric charge

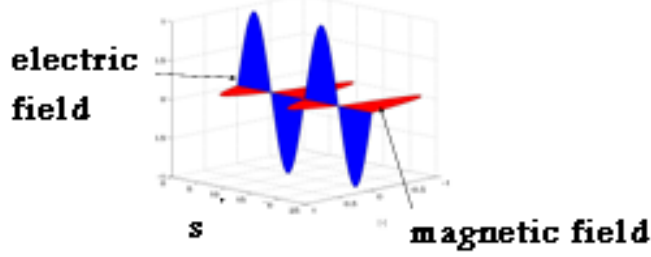

Fig.3. Graphical representation of magnetic flux

\section{Kinetic Energy as a Form of Electromagnetic Waves}

According to the kinetic theory of ideal gases; the average molecular kinetic energy is proportional to the absolute temperature which is expressed by the following equation [15]:

$$
K . E .=\overline{1 / 2 m v^{2}}=3 / 2 k T
$$

where $\boldsymbol{k}$ is the Boltzmann's constant $=1.38 .10^{-23}$ Joules $/ \mathrm{K}$.

Such equation evaluates the kinetic energy of the molecules by its velocity or by thermal energy which is defined by the temperature of the gas confining such molecules. Such relation indicates the common nature of the kinetic energy that activates the motion of particles as the thermal energy which flows as electromagnetic waves. Such similarity was introduced also by Toget [6] who found consistent equations that define the common nature of kinetic and magnetic energies of the flowing electrons. However; the magnetic energy was defined also, according to the followed entropy approach, as a form of electromagnetic waves. Similarly; Rashkov proved also that kinetic energy is identical to electromagnetic energy and that Kinetic energy is conveyed from one object to another object in the form of electromagnetic waves or photons [7]. So; it is possible to postulate that the flow of energy in general is measured by the same unit, Joule, and have a similar nature as flow of electromagnetic waves. 


\section{Similarity of the Flows of Mass and Energy}

The following equations estimate the force $\mathbf{F}$ between two mass particles $\boldsymbol{m}_{\mathbf{1}}, \boldsymbol{m}_{\mathbf{2}}$ and two electric charges $\boldsymbol{Q}_{\mathbf{1}}, \boldsymbol{Q}_{\mathbf{2}}$ separated by distance $\mathbf{r}[1]$ :

$$
\begin{aligned}
& F=\frac{m_{1} m_{2}}{4 \pi r^{2}} \\
& F=\frac{Q_{1} Q_{2}}{4 \pi r^{2}}
\end{aligned}
$$

Reviewing the similarity of the two written equations; it is shown that mass and energy are similar taking into consideration that the electric charge is a form of energy.

Reviewing also the following laws that characterize the mass and energy diffusions as founded by Fick, equation (10), and Fourier, equation (11) [16]:

$$
\frac{\partial C_{A}}{\partial \tau}=D_{A B} \nabla^{2} C_{A}
$$

$\boldsymbol{D}_{\boldsymbol{A B}}$ : Molecular diffusivity

$\boldsymbol{C}_{\boldsymbol{A}}$ : Concentration of component A

$\boldsymbol{\tau}:$ Time

$$
\frac{\partial T}{\partial \tau}=\alpha \nabla^{2} T
$$

$\boldsymbol{\alpha}=$ Thermal diffusivity

$\boldsymbol{k}$ : Thermal conductivity

$\boldsymbol{\rho}:$ Density

$\boldsymbol{c}_{\boldsymbol{v}}:$ Specific heat at constant volume

The similarity of both equations indicates the equivalence of the mechanisms of transport of mass and heat energy. Equivalence of mass and energy is epressed also by the following relativity equation as founded by Einstein [8]:

$$
E=m c^{2}
$$

$\boldsymbol{m}$ : is the mass of the particle which is converted into energy

C: Speed of light

So; the mass and energy are equivalent as expressed by equation (12) and both have similar nature and transport mechanisms as shown in equations (8-11). Accordingly; the flow of mass particles and the flow of energy may be postulated to have a common nature.

\section{Simultaneous Flow of Particles and Waves}

The electrons are supposed to carry electric charge and magnetic moment and it is moving around the nucleus of the atoms. According to the previous analysis; such forms of energy 
have a common nature as electromagnetic waves. So, the electron can be considered as an energized particle whose energy is in the form of electromagnetic waves.

Introducing the following definition of the relativistic kinetic energy $\boldsymbol{K} . \boldsymbol{E}$ [8]:

$$
K . E .=m_{e} c^{2}-m_{0} c^{2}
$$

where $\boldsymbol{m}_{\mathbf{0}}$ is the rest mass of an electron and $\boldsymbol{m}_{\boldsymbol{e}}$ is its relativistic mass. However; such equation lacks other forms of energy that are associated by the flowing electrons, as the electric energy $\boldsymbol{E} . \boldsymbol{E}$ and magnetic energy $\boldsymbol{M} . \boldsymbol{E}$, that should be included in the R.H.S. of equation (13). Such energies are in the form of electromagnetic waves and can be summed into one term $\boldsymbol{E}_{\boldsymbol{w}}$ defined as the total associated electron's wave energy by the following equation:

$$
E_{w}=K \cdot E .+E \cdot E .+M \cdot E
$$

Hence, equation (13) should be modified as follows:

$$
E_{w}=\text { K.E. }+E . E .+M \cdot E=m_{e} c^{2}-m_{0} c^{2}
$$

So; the relativistic electron's mass can be defined as follows:

$$
m_{e}=m_{o}+\frac{E_{w}}{c^{2}}
$$

The rest mass of the electron is found in references as follows [17]:

$$
m_{o}=9.10953 * 10^{-31} \mathrm{~kg} \text {. }
$$

This corresponds to the following amount of particulized or rest energy:

$$
E_{o}=m_{o} c^{2}=8.19 \times 10^{-14} \text { Joules }
$$

Assuming the electron is moving around the nucleus by half the velocity of light; its kinetic energy can be calculated as follows:

$$
K . E .=1 / 2 m_{o} v^{2}=1 / 29.10953 * 10^{-31} *\left(\frac{2.99 * 10^{8}}{2}\right)^{2}=1.016 * 10^{-14} \text { Joule } .
$$

According to the tabulated data of electron's properties [18]; the electron has also an electric energy of $4.1032 * 10^{-14}$ Joule and magnetic energy of $4.0842 * 10^{-14}$ Joule. Substituting such data into equation (14); we find the total wave energy associated by an electron flowing by half the velocity of light as follows:

$$
E_{w}=(1.016+4.1032+4.0842) * 10^{-14}=9.2034 * 10^{-14} \text { Joule }
$$

Hence; the electron's energy has two components; particulized and wave energies. The sum of both components can be found as follows:

$$
E_{e}=E_{o}+E_{w}=(8.19+9.2034) * 10^{-14}=17.3934 * 10^{-14} \text { Joule }
$$


Accordingly; the ratios of the two components to the whole electron's energy $\boldsymbol{E}_{\boldsymbol{e}}$ can be found as follows:

$$
\begin{aligned}
& \mu_{o}=\frac{E_{o}}{E_{e}}=0.472 \\
& \mu_{w}=\frac{E_{w}}{E_{e}}=0.528
\end{aligned}
$$

The values of the ratio of the two components $\boldsymbol{\mu}_{\boldsymbol{o}}$ and $\boldsymbol{\mu}_{\boldsymbol{w}}$ can be considered as the memberships of the flowing electrons to two systems; a system of particulized energy and a system of wave energy. Such values indicate both the energy and mass ratios of the two components of an electron according to the following equations:

$$
\begin{aligned}
& \mu_{o}=\frac{E_{o}}{E_{e}}=\frac{E_{o} / c^{2}}{E_{e} / c^{2}}=\frac{m_{o}}{m_{e}} \\
& \mu_{w}=\frac{E_{w}}{E_{e}}=\frac{E_{w} / c^{2}}{E_{e} / c^{2}}=\frac{m_{w}}{m_{e}}
\end{aligned}
$$

According to the numerically calculated values of the electron's memberships to the two postulated systems; then half of the flowing electron's mass has a wave's nature and the other half has a particle's nature if the electron's velocity is half the velocity of light. So; it is possible to postulate the flow of electrons as a simultaneous flow of particles and waves or a flow of particles activated by the flow of electromagnetic waves. Such postulate explains the dual behavior of the flow of electrons as a flow particles and flow of waves. The proof of such postulate will be verified by reviewing the results of following experiments.

\section{Analysis of Results of the Cathode-Ray Experiment}

During the latter half of the nineteenth century, it was found that while normally dry gases do not conduct an electric current, they do so under very low pressure and then patches of light are seen. Such passage of electricity through gases was studied by a number of physicists, particularly by Faraday, Davy, Crookes and J.J. Thomson [9]. When a current of high voltage $(10,000$ volts $)$ is passed through a gas kept at a very low pressure $(0.01-0.03 \mathrm{~mm} \mathrm{Hg})$, blue rays are seen emerging from the case. These rays were called "Cathode Rays".

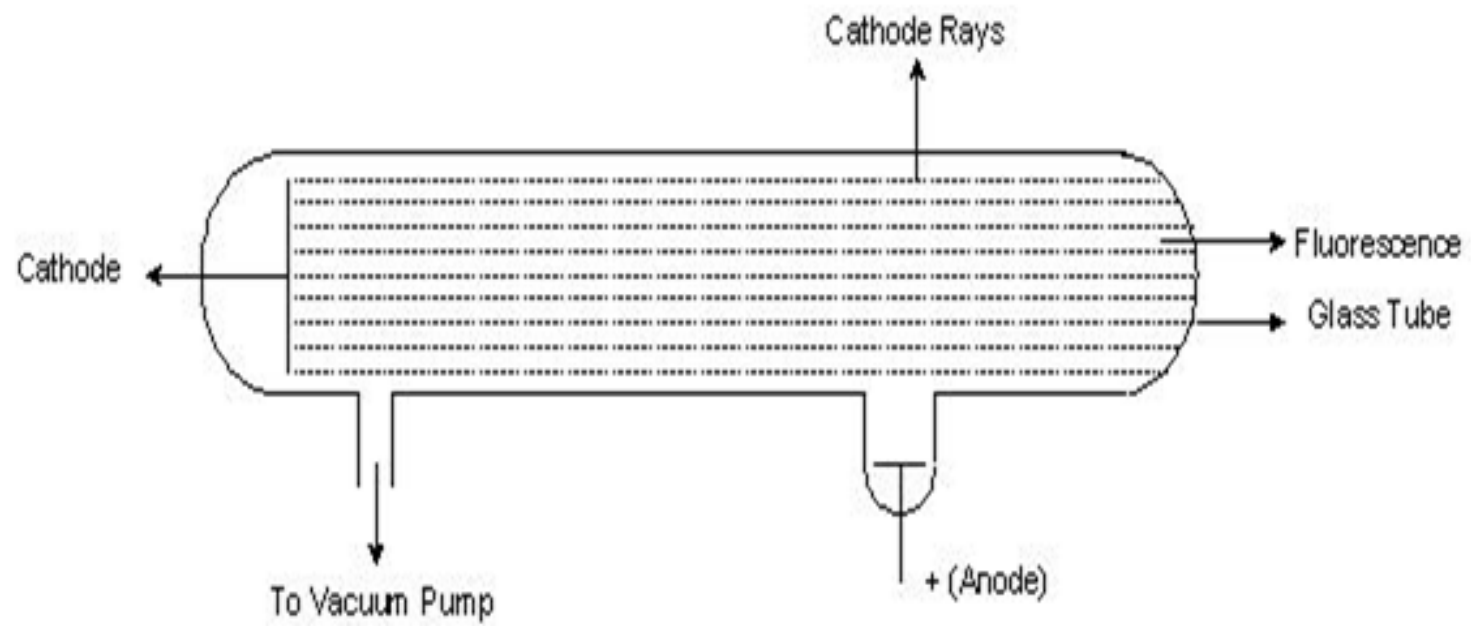

Figure 4. Cathode Rays Experiment 
Reviewing following properties of the cathode rays as found experimentally by J.J. Thomson and others [9]; it will be shown how the postulated simultaneous flow of particles and waves may offer plausible explanations of the found experimental results:

- Cathode rays come out at right angles to the surface of the cathode and move in straight lines as done by the momentum of the particulized component of the flowing electrons.

- Their path is independent on the position of the anode as it is the direction of momentum of the particulized component of the flow of electrons.

- They produce phosphorescence on certain salts like $\mathrm{ZnS}$ and fluorescence on glass which can be done by the wave component of the flowing electrons. .

- They blacken photographic plates as can be done by the wave component of the flowing electrons.

- The rays pass through thin sheet of metals. If the metal sheet is too thick to be penetrated; the rays cast a shadow. Such characteristics may be produced by the electromagnetic waves component of the flowing electrons.

- They produce X-ray when they strike a metal as may be done by both components of the flow of electrons..

- The rays ionize a gas through which they pass as done by the electromagnetic waves component of the flow of electrons that have a negative electric potential.

- They heat a substance on which they fall according to the principles of energy conservation that absorbs the energy component of the flowing electrons and the kinetic energy of the particles.

- They rotate a light wheel placed in their paths. This shows that cathode rays contain material particles having both mass and velocity as done by the particle component of the flow of electrons..

- The mass of a particle present in cathode rays is found to be 1/1837 of H-atom. This shows that the particle is of sub-atomic nature as done by particle component of the flow of electrons..

- Cathode rays are deflected by a magnetic or an electric field showing the particle to be electrically charged, the direction of deflection shows that they are negatively charged as done by electromagnetic waves component of the flow of electrons that have electric and magnetic potentials.

- Cathode rays contain the smallest unit of negative charge in the form of electromagnetic waves of definite negative potential.

- No cathode ray was produced when the tube was completely evacuated as this is a simultaneous flow of particles and energy. The absence of electrons in the confined gas stopped the flow of the two electron's components.

- Different gases produce the same cathode rays as they have the same e/m (charge/mass) ratio. This indicates that the particles present in cathode rays are fundamental constituent of all matter.

So, these electrons and its associated energy represent a simultaneous flow of particles and electromagnetic waves. The ratio $\mathbf{e} / \mathbf{m}$ as found by J. J. Thompson can be considered as $\boldsymbol{\mu}_{\boldsymbol{w}}$ [9]; i.e. the membership of the wave or energy component in the flow of electrons and may be used to find the kinetic energy or velocity of electrons inside the C.R.T.

According to literature [8], light has the nature of particles that releases the electrons from the surface of the cathode. However; such postulate assumes that the particle of light has energy of $\boldsymbol{h} \boldsymbol{v}$ where $\boldsymbol{h}$ is Planck's constant and $\boldsymbol{v}$ is the frequency of light as a wave. Hence, the 
ratio of momentum of normal light photons, that have the wave length $0.55 \mu$, to the momentum of an electron moving at half speed of light, can be calculated as follows:

$$
\frac{(m * v)_{\text {photon }}}{(m * v)_{\text {electron }}}=\frac{m_{\text {photon }}}{m_{\text {electron }}} * \frac{c}{c / 2}=\frac{2.25 * 1.602 * \frac{10^{-19}}{c^{2}}}{9.10953 * 10^{-31}} * 2=0.0000009
$$

Such ratio indicates that it is required more than 1 million of the claimed light particles or photons to bounce one electron. However; it is more plausible to accept the postulate of ionizing the waves to form electric charges or electromagnetic waves of electric potential than to accept the possibility of bouncing an electron with more than a million of such postulated photons. The followed approach deletes the confusion of the light's wave-particle duality as the light is only a wave that may gain an electric potential by the action of an electric field.

\section{De Broglie Waves}

De Broglie speculated in 1924 that light is not the only matter which exhibits the confusing wave-particle duality [12]. He postulated that ordinary "particles" such as electrons, protons, or bowling balls could also exhibit wave characteristics in certain circumstances. Quantitatively, it associated to any particle of mass $\mathbf{m}$ which is moving at velocity $\mathbf{V}$ electromagnetic waves of wavelength $\boldsymbol{\lambda}$ that was found as follows [12]:

$$
\lambda=\frac{h}{m * v}
$$

\section{$\boldsymbol{h}$ : Planck's constant.}

Relatively straightforward tests of equation (19) were elaborated by diffraction and interference; a beam of such "particles" was shown at a diffraction grating and a diffraction pattern of a series of light and dark fringes results [12]. Hence, the wave picture of such postulate was adopted. For electrons, the sizes of slits required for the experiment were of the order of $10^{-11} \mathrm{~m}$ or so, which was readily available.

Such results may be considered as a proof of truth of the postulated nature of kinetic energy as electromagnetic waves and the flow of electrons or any particles as a simultaneous flow of waves and particles. However; the energy of such electromagnetic waves or photons is equal, according to the postulated memberships, to the kinetic energy component of the flowing particles. Hence; such wave's energy can be determined from the kinetic energy according to the following equation [8]:

$$
\sum \frac{\mathrm{h} * \mathrm{c}}{\lambda}=1 / 2 m * v^{2}
$$

Reviewing de Broglie's equation (19), which is verified experimentally, and equation (20), which postulated a wave nature of the kinetic energy of particles, it can be seen that both equations are consistent. According to equations (19) and (20); the wave length of the electromagnetic waves emitted from moving particles is inversely proportional to the mass and velocity of such particles. So; de Broglie hypothesis proves that the flow of particles is a simultaneous flow of particles and electromagnetic waves. 


\section{The Sintering Phenomena}

During sintering; the voids between crystals of some refractory materials, Figure 5, are filled when heat or electric charges pass through the sintered material at temperatures below its melting point $[10,11]$. The mechanism of sintering was considered mainly due to mass diffusion. However; such explanation is not convenient as the diffusing mass may not pass through air-voids, Figure 5, and as sintering is done by applying energy flow at temperatures below the melting point of the sintered materials [10]. According to the introduced postulate that considers the flow of particles as a simultaneous flow of particles and electromagnetic waves; it is possible to consider the flow of heat or electromagnetic waves, during sintering, is simultaneously associated by flow of particles to close the voids between the sintered particles. So, sintering add a proof of the postulated nature of flow of electrons or particles in general as a simultaneous flow of waves and particles.

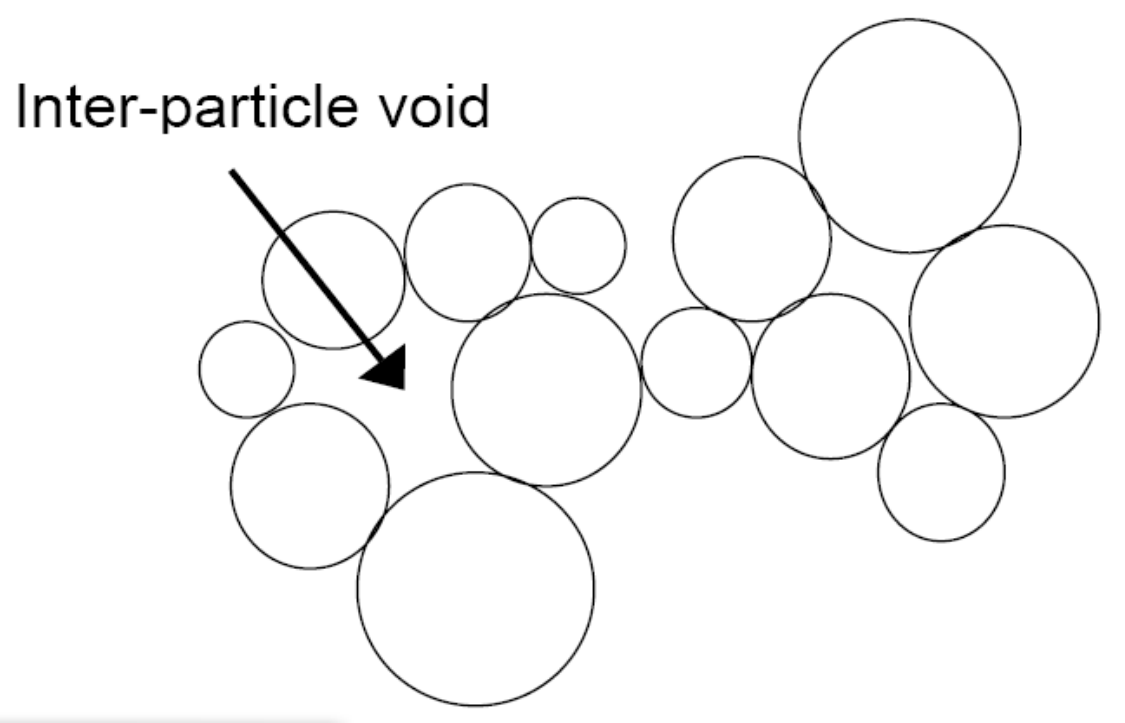

Fig. 5 The voids between sintered particles

\section{Conclusion}

Following an entropy approach that defines the electric charge, magnetic flux and kinetic energy as forms of electromagnetic waves; it was possible to postulate the flow of electrons as a simultaneous flow of electromagnetic waves and particulized energy. According to the same approach; the photoelectric effect is postulated as ionizing reflection of the incident radiation in an electric field. Similarly; the photovoltaic effect is postulated as inducing an electric potential into the incident radiation by the cell's junction. Such postulates clear the confusions in the claimed duality property of particles and light. The truth of such approach is sustained also by finding plausible explanations of the sintering phenomena, de-Broglie hypothesis and thermoelectricity.

\section{References}

[1] R. Haaiday, R. Resnick and J. Walker, Fundamentals of Physics, $7^{\text {th }}$ edition, John Wiley \& Sons, 2004.

[2] D. Rothe, "Space and the Wave-Particle Engima" "Infinite Energy, The Magazine of New Energy Technology”, Vol. 7, 42, 2002, p. 49-57. 
[3] L. Hackermüller, S. Uttenthaler and A. Zeilinger, et al., "The wave nature of biomolecules and fluorofullerenes," Phys. Rev. Lett., 91(9), 90408, 2003.

[4] S. Abdelhady, "A Fundamental Equation of Thermodynamics that Embraces Electrical and Magnetic Potentials" "J. Electromagnetic Analysis \& Applications", March, 2010, 2: pp. 162- 166.

[5] S. Abdelhady, "Thermodynamic Analysis of Electric Charges and Magnetic Flux", Cairo $11^{\text {th }}$ International Conference on Energy and Environment, Ghurgada, Egypt, March, 2009, p.p. 175-185.

[6] C. V. Toget, "The Equivalence of Magnetic and Kinetic Energy," Galilean Electrodynamics, Vol. 16, No. 6., summer 2006, pp. 110

[7] P. Rashkov, "Kinetic energy is identical to electromagnetic energy", Philips-Universtat Marburg, 2010, http://processmodeling.org/theory/physics/kinetic.htm

[8] J. P. Wesley, "Classical Quantum Theory," Chapter 6, Weiherdammstrasse 24, 78176, Blumberg, Germany, 1996.

[9] D. L. Anderson, "The Discovery of the Electron," Princeton, N.J., Van Nostrand, 1964.

[10] M. N. Rahaman "Ceramic processing and sintering," second edition, Marcel Dekker, ISBN: 0-8247-0988-8, 2003.

[11] P. A. Tipler, and A. R. Leweyn, “ Modern Physics.” 4th ed. New York; W. H. Freeman and Co. ISBN 0-7167-4345-0, 2003.

[12] R. D. Rowe, “Thermoelectrics Handbook," Taylor \& Francis. ISBN 0-8493-2264-2, 2006.

[13] A.C. Yunus and A. B. Michael , "Thermodynamics: An Engineering Approach," McGraw-Hill Science Engineering, 2006.

[14] R.L.Liboff, "Kinetic Theory,” Prentice-Hall, Englewood Cliffs, N. J., 1990.

[15] D.M. William, "Analysis of Transport Phenomena," Oxford University Press, 1998.

[16] W.M. Yao, "Review of Particle Physics," Journal of Physics G 33 (1): 77-115, 2006. 